\title{
Comparative approach to ICT practices in civilian and military environments for organizational management during the pandemic crisis
}

\author{
Diana Elena Ranf $^{*}$, Hortensia Gorski ${ }^{1}$, Dorel Badea ${ }^{1}$, and Ioan Virca ${ }^{1}$ \\ 1"Nicolae Balcescu" Land Forces Academy, Military Management Faculty, 3-5 Revolutiei Street, \\ 550170, Sibiu, Romania
}

\begin{abstract}
It is well known that change affects people and implicitly the organizations they belong to, as a place of manifestation and storage of their capabilities. Consequently, the changes also give rise to concerns for at least an acceptable solution to the management of the main changes that occurred in the situation prior to the occurrence of the disruptive events. In this context, the onset of the pandemic crisis puts organizations in front of a fragile reality (relative to the stability of the normal state of functionality of society), fully characterized by volatility, insecurity, complexity and ambiguity. In the search for ideas to improve resilience, there are no predetermined solutions, any method that has registered results in a certain organizational field becoming a candidate of good practice. In this framework, it is proposed the comparative investigation (civilian-military) of ICT practices, as a macro agent of change, used in the field of organizational management during the current pandemic crisis. The approach is based, for each of the two mentioned organizational categories, both on theoretical aspects with hypothetical value identified or derived from the specialized literature specific or complementary to the field in question, and on identified practices. We considered this approach useful because there are enough arguments that the military organization has been a model of organizational-managerial development, which finds wide applicability for business continuity management situations, applicable to civilian organizations. Final expectations are directed, based on data existing so far, towards the identification of flexible and adaptable solutions, with the value of good practice.
\end{abstract}

\section{Functional coordinates of the ICT field in contemporary society}

The term ICT is one that knows obvious conceptual dynamic, imposing essential transformations in all fields of society, some ways of definition highlighting different components and characteristic processes as follows:

\footnotetext{
*Corresponding author: ranfd@yahoo.com
} 
- "Information and communications technology (ICT) refers to all the technology used to handle telecommunications, broadcast media, intelligent building management systems, audiovisual processing and transmission systems, and network-based control and monitoring functions.'[1]

- „Information and communication technology (ICT) refers to both different types of communications networks and the technologies used in them.'[2]

- „Information and communication technology, abbreviated as ICT, covers all technical means used to handle information and aid communication.'"[3]

The social importance of the field is also recognized by the approach initiated and developed by ITU (The International Telecommunication Union / United Nations) which proposed the ICT Development Index as an indicator "to assess and benchmark the developments in information and communication technology (ICT) across countries and over time"[4]. It thus becomes paradigmatic that the development of society 4.0 engages and is based on developments specific to the ICT sector with real implications on how to exercise organizational management, in general, both at the level of management and organizational functions. For the military type organization, the ICT field correlates with the decision-making process (MDMP) in an integrative framework represented by the C4ISR architectural framework.

Today, ICT plays a vital role not only in our personal lives, but also in our organizational life. Digitization, digitalization and digital transformation are not a fad, but rather a necessity, in the context of the digital age, but also of the challenges of the COVID19 pandemic.

\section{ICT practices in the management of civilian organizations during the pandemic crisis}

\subsection{Issues on digital transformation and the COVID-19 pandemic}

The pandemic put organizations face to face with challenges they did not expect and for which many were unprepared. The changes came suddenly, and managers did not have time to plan, prepare and then implement effective strategies. Faced with lockdown policies, some organizations found themselves in a situation where they did not have business continuity plans. Others had, but they did not target the totally unknown aspects of the pandemic, but other categories of threats: power outages, cyber attacks, natural disasters, terrorism, data corruption, etc. As a result, managers found themselves in the situation of making quick decisions, in decision-making universes marked by a very high level of uncertainty. In these cases, organizations, managers, and employees had to adapt, experiment, and identify new ways of working that they had not even thought about before the pandemic.

At the level of governments, public institutions, companies, there was talk of digital transformation that would revolutionize industries and lead to the redefinition of existing businesses and the proliferation of digital ones. However, it should be noted that such a sudden transition was not expected. Companies that had planned multi-year project implementations were forced, in the context of the pandemic, to make the changes in a few weeks or months.

Unfortunately, in Romania the pandemic has caught many organizations unprepared for this chapter related to digital transformation - especially micro-enterprises and small businesses. As a result, switching activities to the online environment has been difficult and has generated many problems and high direct and indirect costs, which, in conjunction with reduced revenues, have led, in many cases, to bankruptcy. In fact, this situation should not 
surprise us if we analyze the Digital Economy and Society Index (DESI) for 2020 Romania, from which we find that Romania ranks 26th among EU countries in terms of digitization, well below the EU average. [5].

\subsection{Teleworking, telecommuting, remote workspace}

A first major challenge in the COVID-19 pandemic was generated by the imposed distancing measures. As a result, organizations around the globe have had to relocate their office staff to their homes in record time. In this context, ICT has become the only way to ensure business continuity. Teleworking, telecommuting and remote work have become words on everyone's lips, with the advent, almost ad-hoc, of virtual offices. Both employees and managers had to reprioritize their work and expectations and adapt very quickly to work remotely or work from home. In the case of the required distance, all meetings with employees and business partners began to take place by teleconference or videoconference. Tools such as videoconferencing and collaboration software were also needed to facilitate communication and coordination. Organizations that thought this was simple were wrong. The creation of a new workspace, which would link all interested entities online, was accompanied by a series of challenges such as: new devices; licensed software installed on multiple computers in employees' homes or other remote locations; password access to software that otherwise could not have been used in any form at home; stable, fast and secure Internet connection; bandwidth / broadband traffic; electronic signatures (e-signature) or admin token for VPN service users; ensuring the security of networks, data and information, etc. Fortunately, organizations that had already implemented remote work systems adapted easier and faster.

Going online has brought to light not only inadequate infrastructure and technology, but also a lack of digital skills. Unfortunately, not all employees knew how to use the new online platforms, nor did they have the skills to use collaboration software effectively. Throughout this period, some organizations have supported their remote support employees to manage issues related to the use of specific ICT tools. However, with or without support, labor productivity has declined in most cases.

\subsection{Internal and external organizational integration through ERP, CRM and SCM systems}

In conditions of an extremely complex and dynamic environment, in order to make quality decisions, managers must analyze an impressive amount of information from both the external and the internal environment. In order to collect, process, analyze and provide quality information at any time, it is necessary to implement in organizations information systems such as Enterprise Resource Planning (ERP) - also called "back-office" systems that integrate all business processes. In this context, the ERP system can be considered as the backbone of any organization. Moreover, in order to be effective, organizations must communicate, collaborate and coordinate their activities both internally - with employees and externally - with customers, suppliers and other business partners. In this sense, in the context of the facilities offered by ICT, organizations must not be limited to internal integration through ERP, but to expand externally - respectively to customers and suppliers. By ensuring the flow of information between all parties involved, "front-office" applications facilitate collaboration with business partners and continuous improvement of relations with them. Customer loyalty is extremely valuable to any organization - regardless of size, field of activity or form of ownership. Given the challenges of the current crisis, companies have had to identify the most effective ways to best serve customers, in the hope that they will not migrate to competition. In this sense, the automation of marketing, the 
provision of sales and post-sales support services through call centers, but also the need for sales forces automation, have imposed the implementation of Customer Relationship Management (CRM) systems and their harmonious integration with Enterprise Resource Planning (ERP) and Supply Chain Management (SCM). As a result, successful organizations combine the strength of an ERP-type "back-office" system with that of CRM and SCM-type "front-office" systems. Practically, at present, most ERP systems have also incorporated CRM and SCM facilities.

During the pandemic, most companies faced sudden decreases or increases in demand and, as a result, had problems with the delivery of raw materials, components, semifinished products, etc. During this period, some companies have chosen to apply the just-intime system to minimize stocks. Supply chain disruptions were the order of the day and their remediation required the identification of effective measures to minimize adverse effects. In this regard, some companies have resorted to various solutions for supply planning, such as SAP (Integrating Business Planning). Also, during this period, companies had the opportunity to take advantage of free access to various platforms, such as the SAP Ariba Discovery platform, which connects customers with suppliers, thus facilitating the purchase of goods and services.

\subsection{Platforms - business models of the 21st century}

Nowadays, more and more customers are willing, not to say they prefer, to have digital experiences. The possibility to integrate on a single platform elements such as: product and / or service presentations, sales operations, marketing teams, market analysis, forecasts, etc., is a challenge and at the same time an opportunity to be closer to stakeholders $24 / 7$. During the pandemic, consumers migrated dramatically to online channels, and as a result, companies were forced to adapt to respond quickly to these changes. At present, the Internet is restructuring the mechanism through which business creates and delivers value. In this sense, platforms are a shift in business design [6] and become the most important business models of the 21 st century [7]. In the last decade we have witnessed a platform revolution [8], as digital networks have become ubiquitous.

Platforms generally connect organizations and people for a common purpose, to make transactions, or to share common resources. Companies such as Google, Apple, Amazon, Facebook, Microsoft have been able to build, with the support of digital platforms, original organizations and self-reinforcing ecosystems [9]. Given the fact that digital networks have become ubiquitous and this trend continues to grow, companies that have the ability to make the most of the strengths and benefits of platforms will be more resilient and better cope with the uncertainties of such a period.

Organizations around the globe can benefit from the facilities of different types of platforms - innovation platforms, transaction platforms, hybrid, stand-alone, parts of a consortium [10]. Digital platforms such as SAP, Apple, IBM, Microsoft, Oracle, Saleforce, Workday, Alibaba, Yahoo, Amazon, Facebook, Google, PayPal, TripAdvisor, LinkedIn, eBay, etc. - are successful examples that have understood the challenges of the new ICT and they quickly integrated them into their business models. Now the platforms are very fashionable and, as a result, many see in them an opportunity that they can take advantage of. This is very good. However, it is important to point out that not all new platforms will be successful businesses in the future. In the context of significant competition in this field, some platform businesses will die, others will survive and others - such as Apple, Microsoft, Facebook, Google - will be key players that will shape the global economy, the future of work and the world politics [11]. Based on years of research and case studies conducted on global organizations such as Amazon, LEGO, Philips, Sneider Electric, etc., 
some experts point out that the design for digital separates winning companies from losing ones [11].

During the pandemic, online transaction platforms were very useful for companies that did not have their own well-developed website or for those that needed logistical support, advertising or online payments. Some companies have resorted to the method of online sales and the advertising of online stores under the pressure of buying changes generated by the crisis. However, from the discussion with the managers we found that many have discovered that this way of selling online has increased their turnover and, as a result, they are interested in continuing this way of working.

\subsection{Migration to Cloud}

With the explosion of data, more and more organizations globally are migrating to Cloud. Using different forms of Cloud services: Infrastructure as a service (Iaas); Platform as a service (Paas) and Software as a service (Saas) (types of Cloud computing services: Infrastructure as a Service (Iaas), Platform as a Service (Paas) and Software as a Service (SaaS), has become a trend all more embraced by organizations. Huge amounts of data are currently being collected and can be analyzed very efficiently using IT tools. Predictive analytics have a major contribution in developing strategies and increasing the quality of decision-making processes. Trends among customers must be anticipated in order to meet them in a timely manner and pleasantly surprise them. Given the fierce competition, a feedforward approach to customers is required, not a feedback approach - as a reaction after they migrate to the competition. This approach is based, among other things, on an in-depth analysis of historical data, combined with new predictive modelling. Big data and data analytics support organizations and their managers to face the challenges related to supply organization, production planning, inventory management (customer management), customer life-cycle management, etc. The merger between cloud computing and analytics has given rise to a new concept - cloud analytics - which has tools for data analysis, processing and manipulation (data analytics, data processing, and data manipulation).

Cloud computing products and services may include: host data warehouses; Cloud based social media analytics; Software as a Service Business Intelligence (Saas BI) etc. [12]. ERP solutions have also begun to migrate to Cloud - as Software as a Service (SaaS). Thus, through the Internet, Cloud ERP facilitates user access, from any location, to ERP applications hosted on different platforms. These Cloud services provide access to hardware and software at lower prices, which makes them very useful especially for small and medium size businesses. In the pandemic situation, for organizations that already had digitized, automated and cloud-based business processes, going through the crisis was much easier. Moreover, some organizations even saw it as an opportunity and took the opportunity to develop and / or reinvent.

\section{ICT practices in the management of the military organization during the pandemic crisis}

The outbreak of the pandemic caused by the Coronavirus infection found military organizations in different regions of the world in a state of functionality characteristic of carrying out specific missions in peace, crisis or war and was at the same time a triggering event for activating processes and procedures dedicated to population in case of civil emergencies. They were involved and acted at NATO level through the following specific structures or mechanisms: NATO's Euro-Atlantic Disaster Response Coordination Center (EADRCC); NATO Support and Procurement Agency (NSPA); Strategic Airlift 
International Solution (SALIS) program [13]. Military institutions also provided logistical, administrative or even managerial support, within the legislative framework, in support of local authorities, depending on the situation and severity of the incidence of pandemic indicators. In a logical operational framework of importance-urgency type, a first measure taken was the resizing or cancellation of certain exercises, at national or international level, which involved large-scale activities in terms of participating forces and technical means, two such examples being Defender-Europe 20 [14] (attended by 37,000 soldiers, including from Romania, and scheduled to start in April and last until May, taking place in seven European countries) and Cold Response 2020 (NATO exercise with approximately 14,000 troops from Norway and 8 other allied nations).

Certainly, from the whole functional spectrum of military-type organizations, the training function (a conceptual equivalent of that of production specific to civilian organizations, according to the general theory of management) was most affected in this pandemic context (still ongoing). A special role in filling the shortcomings presented above has gone to simulation training systems. In this case, as described in Section I, it is about integrated ICT platforms, the solutions (JCATS, VBS, MILES, etc.) widely used in NATO Member States being only an essential component that requires systemic, technical and operational architectural integration. Under the conditions imposed for maintaining social distancing and compliance with specific health rules and for their use, limitations have been imposed (for example in the operational design of the management of the simulation training exercise, from the point of view of the necessary staff expected to be normally involved). Moreover, at the level of security and defense organizations the role of emerging technical systems in the field of ICT is recognized and acknowledged: "With the advent of artificial intelligence and advanced modelling and simulation techniques, tomorrow's crisis management exercises are likely to be more immersive, interactive and dynamic than ever. These technologies will allow future exercises to better replicate real-life parameters and allow for multiple scenarios to play out depending on the decisions taken by participants, thus maximizing their training potential."'[15]

A particularly acceptable situation managed (from the point of view of educational effectiveness) also through ICT platforms / systems is the continuation of specific activities within military institutions of higher post-secondary or high school education. Systems initially provided for e-learning or new systems supported by platforms such as Moodle, Google classroom, Google Meet, etc. were implemented or reactivated, as needed. Official meetings, workshops, conferences were facilitated through video conferencing or webinars. In the field of human resources, especially for the promotion of the military profession, recruitment and selection, which naturally involves face-to-face meetings, the activities also took place via email correspondence (signing, completing and sending specific forms), telephone or videoconferencing. Computer networks with internet or intranet connection are those that also mediated the communication and sending of documents in the daily activities of the commands of military structures.

\section{Discussions and conclusions}

Management, as a science and art, manifests itself through people and for people to achieve a proposed level of performance, depending on the existing organizational capability, based on resources and capabilities. ICT resources transform the situation of an organization in the sense of those previously stated, through specific processes that do not exclude the human presence, this type of resource remaining with strategic status within the managerial culture. One of the observations of professor Oprean, $\mathrm{PhD}$, who stated: "I also notice that in the pandemic, working from home has some advantages for both organizations and employees, but it has considerably weakened relations between colleagues and diminished 
the sense of belonging to the team and weakens the managerial component represented by the value of team management" is also justified in this context. [16]

Due to the deep bureaucratic character of the military type organization and the relative functional and organizational homogeneity, there were premises for uniform and unitary, non-extensive and non-intensive adaptation to the new context of the implementation / use of ICT means to exercise organizational management during the Covid pandemic. Civilian organizations, especially those operating in the business environment, however, have greater organizational-managerial flexibility, which has also manifested itself in adapting the use of ICT to the new organizational context, for different types of industries there are specific and even precise solutions (depending on the location of the production capacities, the specifics of the production or services offered, etc.) of implementation.

Despite the challenges of moving online, most organizations have acknowledged that large-scale digitization has helped them cope with the problems posed by the pandemic. Lockdown made ICT play a much deeper role than it did before the crisis. Unfortunately, some companies lost the battle with the effects of the crisis and died, others survived - but they struggle and manage to "live" only from one day to the next. However, in this sometimes bleak landscape, there were also many resilient, innovative companies, which embraced the new ICT as a catalyst for change.

In the future, quality digital projects are needed, not only based on the implementation of technology, but also on the development of digital skills and digital standards. In this sense, holistic strategies and policies are needed, which harmoniously integrate human resources, technology and processes. The role of management is vital in developing and implementing fluid strategies that synchronize information, processes and human resources. To be successful, a holistic approach to digital transformation is required, with an emphasis on issues such as: redesigning business processes, digital integration of business processes, digital collaboration with suppliers, customers and other business partners.

It is interesting to emphasize the cyber dimension of the use of ICT platforms. For the military organization, specific vulnerabilities and attacks can profoundly affect the fulfillment of the specific mission.

It is also useful to appreciate in crisis conditions, for the educational and training field, the role of MOOC platforms in providing content for training, maintaining or improving skills specific to a profession, a reality still underestimated in normal conditions of functioning of educational systems [17].

The usefulness of the current approach to the article can be proven in terms of taking over good practice, especially in the field of education.

The world is constantly changing, ICT being a catalyst for change. The technological innovations specific to the digital age profoundly affect the way we live and work. Adoption by organizations of new tools, technologies and services specific to digital transformation, such as: digital platforms, Big Data, Internet of Things (IoT), 3-D printing, robotics, artificial intelligence (AI), Machine Learning (ML), Cloud, Blockchain are important elements for increasing organizational resilience. However, it is important to note that these changes are not only related to the desire of organizations, but are largely dependent on the existence of fast and reliable connectivity, which can also be supported by the implementation of the $5 \mathrm{G}$ mobile network. In addition, it should be noted that a sustained and integrated effort is required, which takes into account a number of aspects such as: redesigning business processes, reorganization, readjustment of the methodological-managerial system, acquiring new skills (employee's re-skilling), increasing digital resilience, etc. Adapting to this virtual world and possibly to social distancing will require important changes in organizational culture and leadership style. 


\section{References}

1. http://europeyou.eu/es/what-is-information-and-communication-technology/, accessed on 09.02. 2021

2. oecd-ilibrary.org/science-and-technology/information-and-communication-techno-logyict/indicator-group/english 04df17c2-en, accessed on 09.02.2021

3. ec.europa.eu/eurostat/statistics-explained/index.php/?title=Glossary:Information and communi cation technology (ICT), accessed on 09.02.2021.

4. itu.int/en/ITUD/Statistics/Documents/events/egti2020/IDI2020 BackgroundDocument 20200903.pdf, accessed on 09.02.2021

5. *** European Commission, The Digital Economy and Society Index, p. 14, available at https://ec.europa.eu/digital-single-market/en/digital-economy-and-society-index-desi, accessed on 10.02.2021

6. S.P. Choudary, Platform Scale: How a new breed of start-ups is building large empires with minimum investment. Boston: Platform Thinking Labs (2015)

7. D.B. Yoffie, A. Gawer, M.A Cusumano, A Study of More Than 250 Platforms Reveals Why Most Fail, H. B. R. (2019). Available on https://hbr.org/2019/05/a-study-of-more-than-250platforms-reveals-why-most-fail

8. G.G. Parker, M.W. Alstyne, S.P. Choudary, Platform Revolution: How Networked Markets Are Transforming the Economy — and How to Make Them Work for, W. W. Norton \& Company (2017)

9. L.C. Reillier, B. Reillier, Platform Strategy: how to unlock the power of communities and networks to grow your business, Routledge, (2017)

10. M.A. Cusumano, A. Gawer, D.B. Yoffie, The Business of Platforms: Strategy in the Age of Digital Competition, Innovation, and Power, Harper Business (2019)

11. Ross et.al., Designed for Digital: How to Architect Your Business for Sustained Success, The MIT Press (2019)

12. T. Sanket, Cloud Analytics with Google Cloud Platform, Packt, (2018)

13. *** NATO's Response to the COVID-19 Pandemic, article available at https://shape.nato.int/resources/3/website/0383-20\%20Factsheet\%20COVID-19 en.pdf, accessed on 12.02.2021

14. http://euromil.org/wp-content/uploads/2020/03/Pandemics-and-Military-Readiness.pdf, accessed on 20.02.2021

15. Got Antoine, NATO crisis management exercises: preparing for the unknown, 07 February 2020, article available at https://www.nato.int/docu/review/articles/2020/02/07/nato-crisismanagement-exercises-preparing-for-the-unknown/index.html, accessed on 12.02.2021

16. sites.google.com/view/nl-141/home\#h.i4moffpkkqc, accessed on 10.02.2021

17. Olga Bucovetchi, Radu D. Stanciu, Cristina Petronela Simion, 2016. Study on Designing a Curriculum Suitable for MOOC Platforms Starting Out the Romanian Students' Expectations, Procedia Technology, Volume 22, 2016, Pages 1135-1141 